\title{
Spigelian Port-site Hernia, a Complication after Laparoscopic Cholecystectomy - a Clinical Case Report
}

\author{
Molnar C1, Tîlvescu C1, Neagoe Vl¹, Butiurca VO¹, Molnar CV², Copotoiu C1 \\ ${ }^{1}$ Surgery Clinic 1, County Emergency Clinical Hospital, Tîrgu Mureș, Romania \\ 2 Obstetrics-Gynecology Clinic 1, County Emergency Clinical Hospital, Tîrgu Mureș, Romania
}

\begin{abstract}
Introduction: Spiegelian hernias are rare entities in abdominal wall pathology (2\%). They occur in the semilunar line described by Adriaan van den Spiegel. Klinklosch (1764) defined it as a congenital or acquired defect of the transverse abdominal aponeurosis junction with the Douglas arch. Port-site hernias due to wrong placement of laparoscopic trocars in the right abdominal flank are rare, but possible complications of laparoscopic cholecystectomy.

Case presentation: Our observation shows diagnostic and therapeutic aspects in a patient with port-site Spigelian hernia post laparoscopic cholecystectomy admitted in Surgical Clinic 1, County Emergency Clinical Hospital Tîrgu Mureș in the 28.01.2013 - 30.01.2013 period. Following surgery performed using an open approach, postoperative evolution was favorable, with no signs of recurrence at 9 months postoperatively. Conclusions: Spigelian port-site hernia post laparoscopic cholecystectomy is a very rare entity, iatrogeny being a certainty in its development.
\end{abstract}

Keywords: port-site Spiegelian hernia, laparoscopic cholecystectomy

Received: 2 October 2013

\section{Introduction}

Adriaan van der Spiegel (1578-1625) defines "linea semilunaris" as a transition zone between the navel and the aponeurosis of musculus obliquus internus and of musculus transversus abdominis. It is bordered by the ninth rib cartilage and pubic tubercle, and forms the lateral border of rectus abdominis muscle [1]. This variety of hernia is a congenital or acquired defect of the transverse abdominal aponeurosis at the point of junction between the linea semilunaris and the Douglas semicircular line, Josef Klinklosch (1764) being the first to describe it [2]. The average diameter of the hernial ring varies between 0.5 and 2 $\mathrm{cm}$, and depending on the relationship with the inferior epigastric vascular pedicle, two types of Spigelian hernias are described: upper Spigelian hernia (more frequent) and lower Spigelian hernia (less frequent). The first occurs at the "Spigelian hernia belt" described by Spangen [3] and the second type appears below the virtual line which connects the two anterior superior iliac spines, and they are often misdiagnosed as ascending direct inguinal hernias. Out of all abdominal wall defects, Spigelian hernias account for $1-2 \%$ [3], incarceration occurring only in $20 \%$ of all cases [4].The factors responsible for the development of this type of hernia are: obesity, chronic constipation, ascites, poor tissue quality, multiple pregnancies and peritoneal dialysis [5]. The condition is more common in females (male/female ratio 1:1.18), with a peak incidence in the fifth decade of life [2], and mainly occurs to the right of

Correspondence to: Călin Molnar

E-mail: molnar.calin@gmail.com the umbilicus [6]. The clinical diagnosis is difficult due to uncharacteristic symptoms and signs, often confusing the physician. The treatment is surgical with open or laparoscopic approach, with or without the use of prosthetic materials. Trocar-site hernias are rare complications following laparoscopic cholecystectomy with an incidence of $1.7 \%$, most often being caused by $10 \mathrm{~mm}$ trocars [7]. Potential Spigelian hernias caused by trocar ports are rare [6] and in our observation the parietal defect occurred at the trocar site in the right abdominal flank used for a laparoscopic cholecystectomy.

\section{Case presentation}

We present the case of the patient G.A., aged 60, normostenic, from urban environment, admitted to the emergency service of the Surgery Clinic 1 of the County Emergency Clinical Hospital Tîrgu Mureş, between 28.01.2013 - 30.01.2013, diagnosed with a painful and irreducible incisional hernia. The patient accused abdominal discomfort and constipation, associated with the occurrence of a tumor in the right abdominal flank, which appeared about 2 years ago. Past medical history reveals only a laparoscopic cholecystectomy, which was performed in 2008. The abdominal wall tumor, initially small, progressively increased in size over time and was accompanied in the last 3 months by abdominal pain and altered bowel habits (constipation). On admission the patient presented a subocclusive syndrome with no gas transit, nausea and irreducibility of the right flank tumor formation.

Physical examination revealed an abdomen above the xifo-pubic line with post laparoscopy scars, asymmetri- 


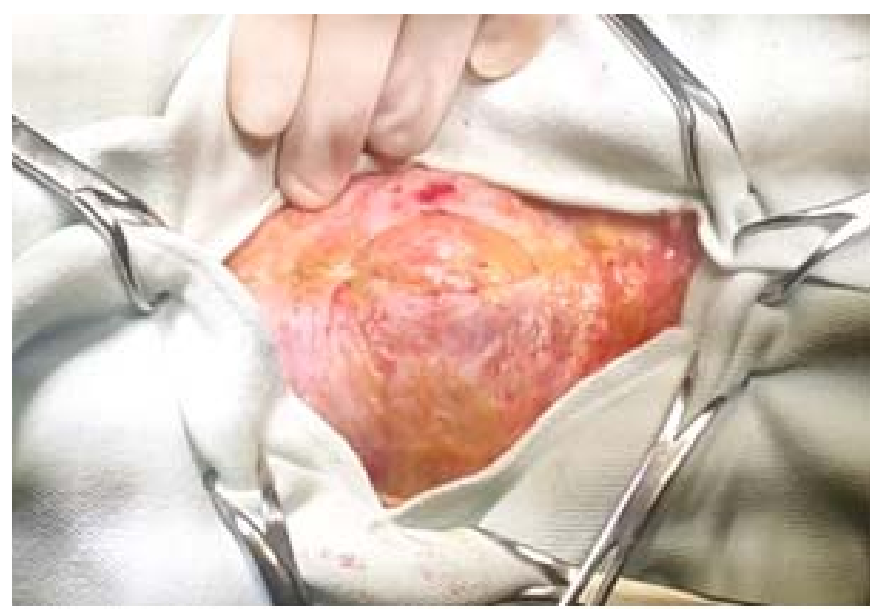

Fig. 1. The hernia sac (after the incision of the external oblique aponeurosis)

cally distorted by the presence of a tumor in the right abdominal flank of about $12 \mathrm{~cm}$ in diameter, with a globular appearance, well defined and centered by a postoperative scar. On palpation we noticed a sensitive parietal tumor of $12 \times 10 \times 5 \mathrm{~cm}$, with pasty consistency, irreducible by taxis, showing a discreet expansion at cough, lateral to the right border of rectus abdominis muscle.

Radioscopic examination revealed a diffuse abdominal aerocholia, with no air-fluid levels, gynecological examination excluded a genital system pathology, and parietal abdominal ultrasound showed a $10 \times 10 \mathrm{~cm}$ tumor with a mixed content, extending from the abdominal cavity through a parararectal abdominal defect.

After a short preoperative preparation the patient underwent surgery in general anesthesia with oro-tracheal intubation. We performed a straight pararectal vertical incision centered on the tumor from the right abdominal flank. The aponeurosis of the abdominal external oblique muscle highlights the presence of a tumor, $10 \mathrm{~cm}$ in diameter, that pushes the aponeurotic structures upwards. After cutting the aponeurosis we spotted a hernia sac of $10 \times 8 \mathrm{~cm}$ (Figure 1).

We isolated the sac up to the parietal breach and we found a $3 \mathrm{~cm}$ diameter parietal defect with fibrous edges,

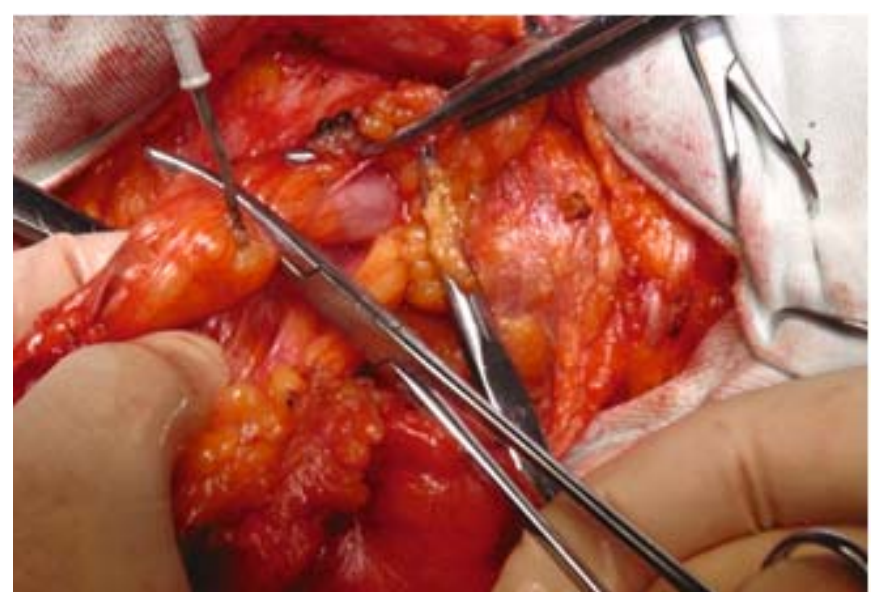

Fig. 3. The aspect of the hernial sac after cheliotomy and its content

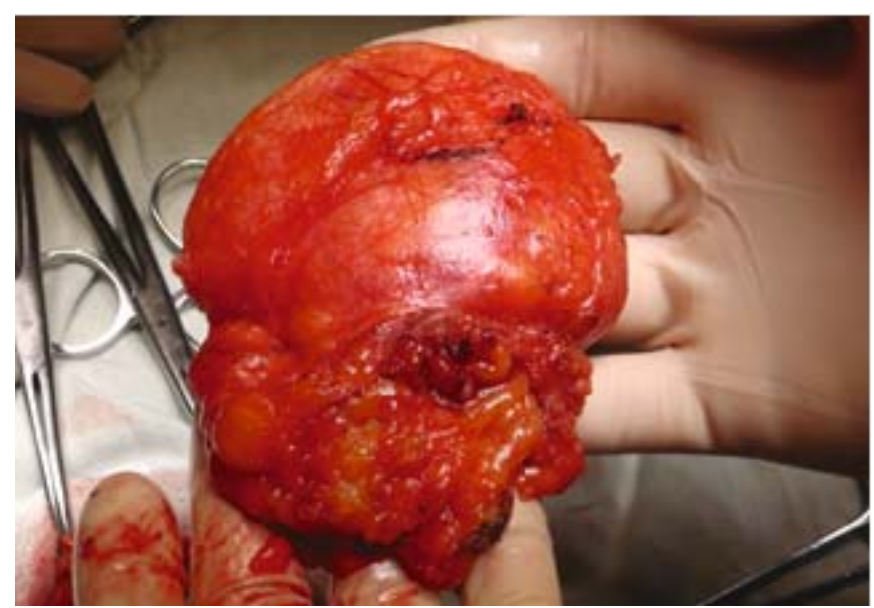

Fig. 2. The hernia sac with the adherent omentum

located at the level of Spigel's crescent line. Herniotomy was performed and the great omentum appeared to be adherent to the sac (Figure 2). We performed partial resection of the great omentum along with the hernial sac (Figure 3).

After the resection of the peritoneal excess, we detected a $3 \mathrm{~cm}$ parietal defect located on the semilunar line, most likely produced by one of the trocars used previously for the laparoscopic cholecystectomy (Figure 4).

Given the good quality of the muscular wall, the absence of other risk factors and the occurrence of this condition in the context of a iatrogenic factor (trocar placement through the Spangen strip), we considered appropriate to perform the abdominal wall plasty with autologous material. We closed the hernia ring with slowly absorbable threads (Figure 5) and then we performed a parietal splitting using the external oblique muscle's aponeurosis (Figure 6).

The immediate postoperative evolution was favorable (discharge of the patient on postoperative day 2), no signs of relapse at 9 months.

\section{Discussions}

Spiegelian hernia is a rare entity $(1-2 \%$ of all abdominal wall hernias) $[3,6]$, about 1000 cases being quoted in literature [6]. In the casuistry of the past 10 years of the Surgery Clinic 1 of the County Emergency Hospital Tîrgu Mureş,

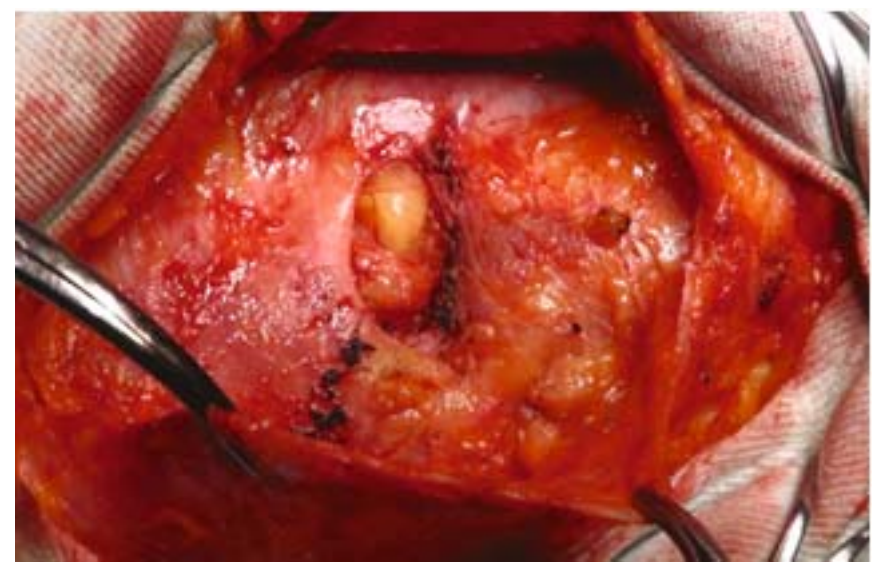

Fig. 4. The parietal defect located at the crescent line 


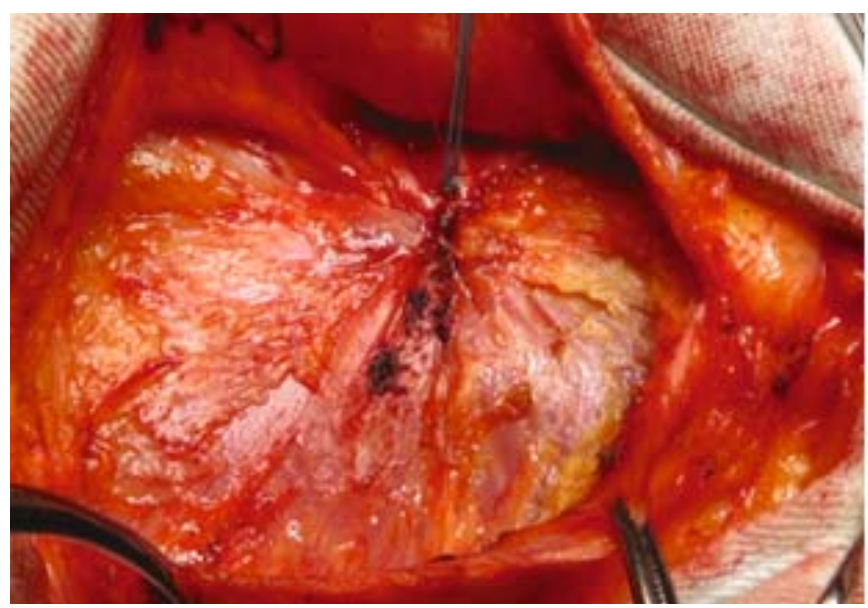

Fig. 5. Closing the parietal defect with slowly resorbable threads

only five patients ( 4 females and 1 male) were hospitalized and surgically treated for Spigelian hernias.

Laparoscopic trocar-site hernias are rare $(1.7 \%)$ and the ports made by $10 \mathrm{~mm}$ trocars are most incriminated $[7,8]$. In the literature very few cases of incisional hernias are described, after using 3-5 $\mathrm{mm}$ trocars, as shown by Bunting in a meta-analysis of 7 studies involving a total of 5984 patients [7]. [8]:

Tonouchi classified trocar-site hernias into three types

- Early-onset type: dehiscence of the anterior fascial plane, posterior fascial plane, and peritoneum. They begin to develop in the early stages after surgery, often presenting as a small-bowel obstruction.

- Late-onset type: dehiscence of the anterior fascial plane and posterior fascial plane. In this type of hernia the peritoneum constitutes the hernia sac. They have been recognized, in many large series, to be related to complications of the trocar insertion and almost always develop in the late stages several months after surgery.

- Special type: dehiscence of the whole abdominal wall with the protrusion of the intestines and other tissues.

If the trocars are placed between the crescent line laterally and the rectus abdominis muscle medially, at the level of the semilunar line or close to it, Spigelian port-site hernias may result as the literature shows [6]. This paper wishes to warn as to the risk of port-site hernia after inadvertent placement of small laparoscopic trocars through a herniar region (Spangen's hernia belt). Our observation describes a 60 year-old normostenic patient, with a history of laparoscopic cholecystectomy, where a late-onset type Spigelian trocar-site hernia was a result of wrong laparoscopic port placement in the right abdominal flank. Only one case of similar hernia has been recorded in the literature [6], the etiology and evolution of this particular condition being a diagnostic challenge.

Showing few symptoms and often with few specific signs, Spigelian hernia is difficult to diagnose. Complete

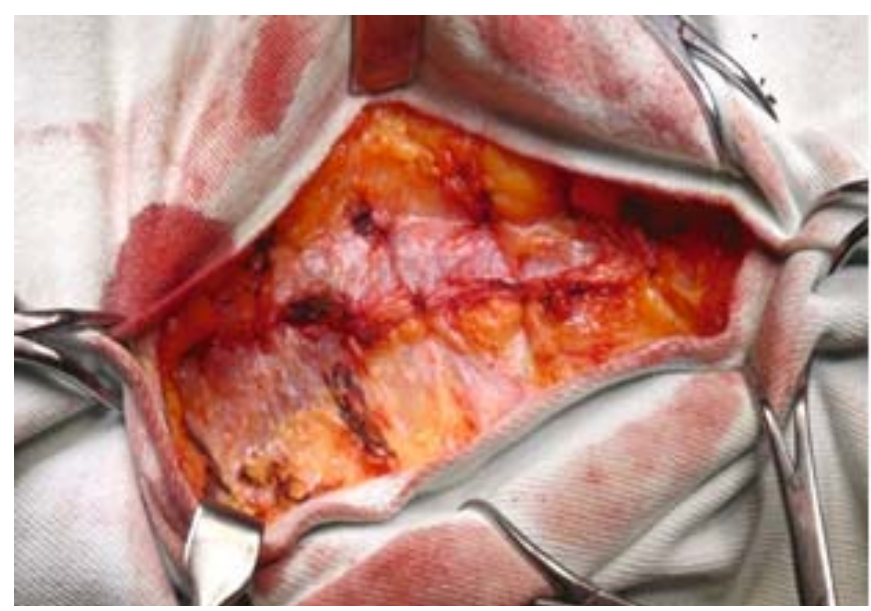

Fig. 6. Parietal splitting using the external oblique muscle's aponeurosis

clinical examination and imagistic examinations (ultrasound, computer tomography) are sufficient in $86 \%$ of the cases [9]. The differential diagnosis of this type of hernia includes pathology of the abdominal wall (soft tissue tumors, hematomas) and intraperitoneal pathology (tumors, ectopies, inflammatory digestive and gynecological conditions). If incarcerated hernia occurs, the diagnosis becomes a surgical emergency, as the situation encountered in the present case.

The treatment of choice is surgical, with multiple ways of approach (classical or by laparoscopic methods) and multiple wall defect closure methods. The recurrence rate after open approach (Bachy technique) is about $4 \%$ [11], while in the intraperitoneal laparoscopic approach (Carter technique) or totally extraperitoneal (Moreno-Egea technique) is almost zero [12]. Procedures that use prosthetic material (Flament technique) bare the inconvenience of introducing heterologous material and therefore the risk of foreign body reactions. In the presented case, we performed a classical approach with autologous material, on a normostenic patient without associated pathologies, with a good biological profile, pertaining that the hernia was irreducible and painful.

\section{Conclusions}

Spigelian hernias are a rare variety of hernias, the diagnosis being established through general clinical examination for uncomplicated large hernias, imagistic investigations (ultrasound, computed tomography) being useful only for complicated hernias. The possibility of trocar-site Spigelian hernias after laparoscopic interventions requires attention upon trocar placement in a herniar region of the abdominal wall. The treatment of choice is surgical, the prosthetic materials are justified only if there are other risk factors.

\section{References}

1. Schwartz SI, Ellis $H$ (eds). Maingot's abdominal operations. 9th ed. Norwalk: Appleton \& Lange. 1990, vol. 1, ch. 11, p. 269.

2. Houlihan TJ. A review of Spigelian hernias. Am J Surg 1976;131(6):734-5. 
3. Spangen L. Spigelian hernia. World J Surg. 1989:13(5):573-80.

4. Artioukh DY, Walker SJ. Spigelian herniae:presentation, diagnosis and treatment. J R Coll Surg Edinb. 1996;41(4):241-3.

5. Torres K, Chrościcki A, Torres A, et al. Spiegelian hernia - anatomy, diagnosing and imaging difficulties: a report of 2 cases. Folia Morphol. 2009;68(3):179-183.

6. Mahdi SA, Jawad M, Nazir A, Naroo GY. Spigelian hernia: a complication of laparoscopic cholecystectomy. Hong Kong Journal of Emergency Medicine, 2010;17(4):388-391

7. Bunting DM. Port-site hernia following laparoscopic cholecystectomy JSLS: Journal of the Society of Laparoendoscopic Surgeons. 2010;14(4): 490 .
8. Tonouchi H, et al. Trocar site hernia. Archives of Surgery. 2004;139(11): 1248.

9. Testa T, Falco E, Celoria G, et al. Spigelian hernia: its echotomographic diagnosis and surgical treatment. [Italian] G Chir. 1992;13(1-2):29-31.

10. Khurshid N, Chung M, Horrigan T, Manahan K, Geisler JP. 5-Millimeter Trocar-Site Bowel Herniation Following Laparoscopic Surgery. JSLS: Journal of the Society of Laparoendoscopic Surgeons. 2012;16(2):306.

11. Margaritescu D, Cartu D. Surlin V, et al. Hernia Spiegel. Experiența proprie. Revista literaturii. Craiova Medicala. 2008;10(3):161-164.

12. Moreno-Egea A, Carrasco L, Girela E, et al. Open vs laparoscopic repair of spigelian hernia: a prospective randomized trial. Archives of surgery. 2002;137(11):1266 